\title{
Metzlerian and Generalized Metzlerian Matrices: Some Properties and Economic Applications
}

\author{
Giorgio Giorgi ${ }^{1} \&$ Cesare Zuccotti $^{1}$ \\ ${ }^{1}$ Department of Economics and Management, Via S. Felice, 5 - 27100 Pavia, Italy \\ Correspondence: Giorgio Giorgi, Department of Economics and Management, Via S. Felice, 5 - 27100 Pavia, Italy. \\ E-mail: ggiorgi@eco.unipv.it
}

Received: February 19, 2015 Accepted: March 3, 2015 Online Published: March 25, 2015

doi:10.5539/jmr.v7n2p42 URL: http://dx.doi.org/10.5539/jmr.v7n2p42

\begin{abstract}
In the first part of the paper we consider the main properties, with respect to stability and existence of solutions of multi-sectoral economic models, of Metzlerian and Morishima matrices. In the second part we introduce various generalized Metzlerian matrices, in order to enlarge the results of Ohyama (1972) in the study of stability and comparative statics for a Walrasian-type equlibrium model.
\end{abstract}

Keywords: Metzlerian matrices, Morishima matrices, Walrasian system, stable matrices

\section{Introduction}

Metzlerian matrices or Metzler matrices (in honor of the American economist L. A. Metzler (1913-1980)) are square (real) matrices in which all the off-diagonal elements are nonnegative: $a_{i j} \geqq 0, \forall i \neq j$. Following the terminology of Fiedler and Pták (1962) we can say that a square matrix $A$ is a Metzlerian matrix if and only if $-A \in \mathcal{Z}$, where $\mathcal{Z}$ is the class of square matrices such that $a_{i j} \leqq 0, \forall i \neq j$. Therefore, the relevant properties of Matzlerian matrices can be derived from the "parallel" properties of the $\mathcal{Z}$-matrices, i. e. of the square matrices belonging to the $\mathcal{Z}$-class. In Linear Algebra Metzlerian matrices are sometimes called essentially nonnegative matrices or also quasi-positive matrices. These matrices appear in several applications, especially in economic analysis, as they translate the assumption of gross substitutability in the study of stability (local and global) of the equilibrium solution of a Walrasian economic model of general equilibrium.

If we denote by $e_{i}\left(p_{1}, \ldots, p_{n}\right), i=1, \ldots, n$, the $n$ excess demand functions for the $n$ goods exchanged in a Walrasian market, where $p_{i}$ is the price of the $i$-th good, the $j$-th good is a gross substitute (respectively a weak gross substitute) for the $i$-th good, if for the Jacobian matrix $J e(p)$ we have

$$
\begin{gathered}
e_{i j} \equiv \partial e_{i} / \partial p_{j}>0, \forall i \neq j \\
\left(e_{i j} \equiv \partial e_{i} / \partial p_{j} \geqq 0, \forall i \neq j\right) .
\end{gathered}
$$

This notion was introduced in economic analysis by Mosak (1944) and by Metzler (1945) and subsequently widely used in the study of the uniqueness of solutions in general equilibrium theory, in stability analysis of the same, in comparative statics analysis (Hicksian Laws, Le Chatelier-Samuelson Principle) and in other economic and noneconomic subjects: see, e. g., Arrow and Hahn (1971), Bermann, Neumann and Stern (1989), Farina and Rinaldi (2000), McKenzie (2002), Morishima (1964), Murata (1977), Nikaido (1968), Takayama (1985), Woods (1978).

This paper is structured as follows.

In Section 2 we give an overview of the main properties of Metzlerian matrices and of Morishima matrices.

In Section 3, by introducing new classes of "generalized Metzlerian matrices" we perform a generalization of some results of Ohyama (1972) on stability and comparative statics for a Walrasian-type economic system.

In this paper we adopt the following conventions:

- $N=\{1,2, \ldots, n\}$ is the set of the first $n$ positive integers.

- Unless otherwise stated, all matrices and vectors are real. 
- $\quad[0]$ denotes the matrix or the vector with all zero entries, $A_{i}$ denotes the $i$-th row of the matrix $A, A^{j}$ denotes its $j$-the column.

- $u$ is the vector whose entries are all $1 . \quad u^{1}=[1,0, \ldots, 0]^{\top}, \quad u^{2}=[0,1, \ldots, 0]^{\top}, \ldots$, $u^{n}=[0,0, \ldots, 1]^{\top}$.

- By $A_{k k}$ we denote the $k$-th principal minor of order $(n-1)$ of the square matrix $A$, of order $n>1$.

- If $A$ and $B$ are matrices of the same order $(m, n)$, the notations $A \geqq B, A \geq B, A>B$ stand, respectively, for: $a_{i j} \geqq b_{i j}, \forall i, j ; A \geqq B$ but $A \neq B ; a_{i j}>b_{i j}, \forall i, j$. When $B=[0]$, this means that $A$ is, respectively, a nonnegative, a semipositive, a positive matrix. The same convention is used to compare two vectors of the same dimension. The notations $A \leqq B, A \leq B, A<B$ are obvious.

- $\mathfrak{D}$ is the set of diagonal matrices; $\mathfrak{D}_{+} \subset \mathfrak{D}$ is the proper subset of $\mathfrak{D}$ of matrices with a positive diagonal.

- $\rho(A), A$ square, is the spectral radius of $A$, whereas $\lambda^{*}(A)$ is the Perron-Frobenius root of $A \geqq[0]$.

- A square matrix $A$ of order $n$ has a row dominant diagonal (in the sense of McKenzie (1960)) if there exist numbers $d_{i}>0, i=1, \ldots, n$, such that

$$
d_{i}\left|a_{i i}\right|>\sum_{j \neq i} d_{j}\left|a_{i j}\right|, i=1, \ldots, n .
$$

If, in addition, $a_{i i}<0, i=1, \ldots, n$, then $A$ has a negative row dominant diagonal. It can be proved (McKenzie (1960)) that if $A$ has a row dominant diagonal, then $A$ has a column dominant diagonal, that is, there exist numbers $d_{j}>0, j=1, \ldots, n$, such that

$$
d_{j}\left|a_{j j}\right|>\sum_{i \neq j} d_{i}\left|a_{i j}\right|, j=1, \ldots, n .
$$

Also the converse holds; therefore it is convenient to speak of "dominant diagonal", omitting "row" or "column".

McKenzie (1960) introduced also the notion of a matrix $A$ with a quasi-dominant diagonal (see also Kemp and Kimura (1978), Uekawa (1971, footnote 5) and Mckenzie (2002)). A square matrix $A$ of order $n$ has a quasidominant diagonal if there exist numbers $d_{i}>0$ such that

$$
d_{i}\left|a_{i i}\right| \geqq \sum_{j \neq i} d_{j}\left|a_{i j}\right|, i=1, \ldots, n,
$$

with at least one strict inequality which must hold

a) for at least one index $i \in N=\{1,2, \ldots, n\}$, if $A$ is indecomposable or irreducible, i. e. there does not exist a subset $L \neq \varnothing$ in $N$ such that $(i \in L, j \notin L) \Longrightarrow a_{i j}=0$;

b) for at least one index $i \in L, L$ being any subset of $N$, if $A$ is decomposable (i. e. not indecomposable).

McKenzie (1960) proves that $A$ has a quasi-dominant diagonal if and only if it has a dominant diagonal.

A basic result on dominant diagonal matrices is: every matrix having a dominant diagonal is non-singular.

- A square matrix $A$ (not necessarily symmetric) is said to be quasi-negative definite if $x \neq[0]$ implies $x^{\top} A x<0$. (If $A$ is symmetric the previous inequality characterizes the negative definite matrices).

\section{Metzlerian and Generalized Metzlerian Matrices}

We have already recalled in the Introduction that $A$, real and square, is Metzlerian if every off-diagonal element of $A$ is nonnegative, i. e. if $-A \in \mathcal{Z}$, in the terminology of Fiedler and Pták (1962). Therefore, all the characterizations of the so-called $\mathcal{K}$-class of square matrices (always in the terminology of Fiedler and Pták), more usually called $\mathcal{M}$ class, can be transferred to the Metzlerian class. For a survey of the characterizations of the $\mathcal{K}$-class (or $\mathcal{M}$-class) see Fiedler and Pták (1962), Berman and Plemmons (1976), Plemmons (1977), Magnani and Meriggi (1981), Poole and Boullion (1974).

We give in the following theorem only a partial overview of the results on Metzlerian matrices, obtained from the corresponding results on $\mathcal{K}$-matrices.

Theorem 1. Let $A$ be a square $n$ by $n$ matrix with $a_{i j} \geqq 0, \forall i \neq j$ (i. e. $A$ is a Metzlerian matrix). Then the following conditions are mutually equivalent. 
i) There exists an $x \geq[0]$ such that $A x<[0]$.

ii) For any $c \leqq[0]$, there exists an $x \geqq[0]$ such that $A x=c$.

iii) The matrix $A$ is non-singular and it holds $A^{-1} \leq[0]$.

iv) The leading principal minors of $A$ alternate in sign, beginning with the negative sign:

$$
a_{11}<0,\left|\begin{array}{ll}
a_{11} & a_{12} \\
a_{21} & a_{22}
\end{array}\right|>0, \ldots,(-1)^{n} \operatorname{det}(A)>0 .
$$

v) The $\left(\begin{array}{l}n \\ k\end{array}\right)$ principal minors of order $k$ of $A$ have the sign of $(-1)^{k}, k=1, \ldots, n$. In other words, $A$ is Hicksian (see, e. g., Kemp and Kimura (1978)).

vi) Each characteristic root of $A$ has a negative real part: $\operatorname{Re}\left(\lambda_{i}\right)<0, \forall i$. In other words, $A$ is stable or negative stable.

vii) If $A$ is written in the form $A=[B-\mu I]$, with $B \geqq[0]$ and $\mu \in \mathbb{R}$, any of the conditions of the present theorem is equivalent to: $\mu>\lambda^{*}(B)$, where $\lambda^{*}(B)$ denotes the Frobenius characteristic root or dominant characteristic root of $B$ (see, e. g., Berman and Plemmons (1976) and the next Theorem 5).

viii) $A$ has a negative dominant diagonal.

ix) There exists a positive definite (symmetric) matrix $H$ such that $H A$ is quasi-negative definite (i. e. $x \neq$ [0] implies $\left.x^{\top} H A x<0\right)$.

We have seen that a Metzlerian matrix is stable if and only if any one of the previous conditions $i$ )-ix) of Theorem 1 holds. Now we want to better focalize the role of the Metzlerian assumption in the analysis of stability (see, e. g., Giorgi (2003), Kemp and Kimura (1978), Newman (1959), Murata (1977), Hershkowitz (1992), Hale and others (1999)). We recall first the following definitions.

Definition 1. Let $A$ be a (real) square matrix.

a) The matrix $A$ is said to be stable (or negative stable) if every characteristic root of $A$ has its real part negative: $\operatorname{Re}\left(\lambda_{i}\right)<0, \forall i$.

b) The matrix $A$ is said to be $D$-stable if $D A$ is stable for every diagonal matrix $D \in \mathfrak{D}_{+}$.

c) The matrix $A$ is said to be totally stable if every principal submatrix of $A$ is $D$-stable.

d) The matrix $A$ is said to be $S$-stable if $S A$ is stable for every (symmetric) positive definite matrix $S$.

e) The matrix $A$ of order $n$ is said to be Hicksian (in honor of the English economist J. Hicks (1904-1989)), if all of its principal minors of order $r$ have the sign of $(-1)^{r}, r=1, \ldots, n$. In the mathematical literature Hicksian matrices are also called (NP)-matrices. The matrix $A$ is said to be imperfectly Hicksian (see, e. g., Gandolfo (2010), Takayama (1985)) if every its principal minor of order $(n-1)$ has the sign of $-\operatorname{det}(A)$.

f) Let $A$ and $B$ be any two real square matrices of the same order. The matrix $B$ is said to be sign-similar to $A$ if the sign pattern $(-,+, 0)$ of $B$ is the same as the sign pattern of $A$, regardless of the magnitudes of the entries in $A$ or $B$. Let $Q_{A}$ denote the set of square matrices sign-similar to a given matrix $A$. Then $A$ is said to be sign-stable or qualitatively stable if and only if every member of $Q_{A}$ is stable.

The results contained in the following theorem are well-known (see the previously quoted authors).

Theorem 2. Let $A$ be a (real) square matrix of order $n$. Then:

1) If $A$ has a negative dominant diagonal, then $A$ is $D$-stable and Hicksian.

2) $A$ is stable if and only if there exists a positive definite matrix $H$ such that $\left(H A+A^{\top} H\right)$ is definite negative. This is a well-known result of Lyapunov on the stability of matrices. 
3) If $A$ is symmetric, then we have the following equivalences

$$
\{A \text { is Hicksian }\} \Longleftrightarrow\{A \text { is negative definite }\} \Longleftrightarrow\{A \text { is stable }\} .
$$

4) If $A$ is quasi-negative definite, then $A$ is totally $S$-stable and therefore totally stable, $S$-stable, $D$-stable and stable. Moreover, $A$ is Hicksian.

5) If $A$ is totally stable, then $A$ is $D$-stable (and obviously $A$ is stable).

6) If $A$ is qualitatively stable, then $A$ is $D$-stable (and obviously $A$ is stable); moreover, if it holds $a_{i i}<0$, $\forall i=1, \ldots, n$, then if $A$ is qualitatively stable, then $A$ is totally stable.

If we assume that $A$ is Metzlerian, besides the equivalences described in Theorem 1, we get also the equivalences contained in the following theorem (see Kemp and Kimura (1978), McKenzie (1960), Newman (1959), Giorgi (2003)).

Theorem 3. Let $A$ be Metzlerian; then the following equivalences hold

$$
\begin{gathered}
\{A \text { is totally stable }\} \Longleftrightarrow\{A \text { is } D \text {-stable }\} \Longleftrightarrow\{A \text { is totally stable }\} \Longleftrightarrow \\
\Longleftrightarrow\{A \text { is Hicksian }\} \Longleftrightarrow\{A \text { has a negative dominant diagonal }\} \Longleftrightarrow \\
\Longleftrightarrow\{\text { any one of the conditions of Theorem } 1 \text { holds }\} .
\end{gathered}
$$

Arrow and McManus (1958) introduced the following definition of $D$-stable matrices, we may call "strong $D$ stability".

Definition 2. The square matrix $A$ is said to be strongly $D$-stable if

$$
D \in \mathfrak{D} \Longrightarrow\left\{D A \text { is stable } \Longleftrightarrow D \in \mathfrak{D}_{+}\right\} .
$$

The said authors proved the following result:

Theorem 4. (Arrow and McManus (1958)). If there exists $E \in \mathfrak{D}$ such that $E^{-1} A E$ is Metzlerian and stable or it is quasi-negative definite, then $A$ is strongly $D$-stable.

Corollary 1. Let $A$ be Metzlerian and stable. Then $A$ is strongly $D$-stable.

Following Karlin (1959), we note that Metzlerian matrices may also be treated as a special transformation of nonnegative (square) matrices, since by adding a sufficiently large positive multiple of the identity matrix $I$, we can write, with $A$ Metzlerian,

$$
C=A+\mu I,
$$

where $C \geqq[0]$. It is therefore possible to obtain, for Metzlerian matrices, Perron-Frobenius-type results (see. e. g. Arrow (1989), Kemp and Kimura (1978), Karlin (1959), Berman and Plemmons (1976)).

Theorem 5. Let $A$, of order $n$, be Metzlerian. Then $A$ has a characteristic root $\lambda^{*}(A)$ (Frobenius root or dominant characteristic root) such that:

i) $\lambda^{*}(A)$ is real, it is the largest real characteristic root of $A$; moreover, $\lambda^{*}(A) \geqq \operatorname{Re}(\lambda)$, being $\lambda$ any other characteristic root of $A$.

ii) With $\lambda^{*}(A)$ there is associated a semipositive characteristic vector $x^{*} \geq[0]$.

iii) It holds $[\mu I-A]^{-1} \geq[0]$ if and only if $\mu>\lambda^{*}(A), \mu \in \mathbb{R}$.

iv) $\lambda^{*}(A) \geqq 0$ if and only if there exists a vector $x \geq[0]$ such that $A x \geqq[0]$.

If we assume that $A$ is also indecomposable, the previous theorem can be strengthened.

Theorem 6. Let $A$, of order $n$, be Metzlerian and indecomposable. Then:

i) The characteristic vector $x^{*}$ associated to $\lambda^{*}(A)$ can be chosen positive; no other characteristic vector associated to characteristic values $\lambda$ different from $\lambda^{*}(A)$ has this sign property. 
ii) It holds $[\mu I-A]^{-1}>[0]$ if and only if $\mu>\lambda^{*}(A), \mu \in \mathbb{R}$.

iii) $\lambda^{*}(A)$ is a simple root of the characteristic equation of $A$.

iv) $\lambda^{*}(A)>0$ if and only if there exists a vector $x \geq[0]$ such that $A x>[0]$.

Metzlerian matrices also arise in the study of positive dynamic systems (see, e. g., Farina and Rinaldi (2000)): if we consider the linear system of differential equations

$$
x^{\prime}(t)=A x(t), \quad x([0]) \in \mathbb{R}^{n}
$$

with $t \geqq 0$ and with solution

$$
x(t)=e^{A t} x([0]),
$$

where $e^{A t} \equiv \sum_{k=0}^{+\infty} \frac{t^{k}}{k !} A^{k}$, it can be proved (see, e. g., Minc (1988)) that $e^{A t} \geqq[0]$ if and only if $A$ is Metzlerian.

A generalization of Metzlerian matrices, which has some importance in economic analysis, is given by the so-called Morishima matrices (Morishima (1952, 1970)).

Definition 3. A square matrix $A$ of order $n$ is a Morishima matrix if there exists a permutation matrix $\Pi$ such that

$$
\Pi A \Pi^{-1}=\left[\begin{array}{ll}
A_{11} & A_{12} \\
A_{21} & A_{22}
\end{array}\right]
$$

where $A_{11}$ and $A_{22}$ are square and Metzlerian and $A_{12} \leqq[0], A_{21} \leqq[0]$.

Morishima (1952) requires the stronger condition that $A_{11}$ and $A_{22}$ are to be square and nonnegative. Moreover, he proves that if $a_{i j} \neq 0, \forall i, j=1, \ldots, n$, then this stronger requirement is equivalent to: $a_{i i}>0, \forall i$; sign $a_{i j}=\operatorname{sign} a_{j i}$, $\forall i \neq j$; sign $a_{i j}=\operatorname{sign} a_{i k} a_{k j}$ for any $i, j, k$ distinct.

Taking into account that it holds

$$
\left[\begin{array}{cc}
I & {[0]} \\
{[0]} & -I
\end{array}\right]\left[\begin{array}{ll}
A_{11} & A_{12} \\
A_{21} & A_{22}
\end{array}\right]\left[\begin{array}{cc}
I & {[0]} \\
{[0]} & -I
\end{array}\right]=I^{*} A I^{*}=\left[\begin{array}{cc}
A_{11} & -A_{12} \\
-A_{21} & A_{22}
\end{array}\right],
$$

we can derive properties of a Morishima matrix form those of the corresponding Metzlerian matrix or of the corresponding nonnegative matrix. In particular, we have the following results concerning, respectively, stability and Perron-Frobenius properties of a Morishima matrix.

Theorem 7. Let $A$ be a Morishima matrix; then the equivalences of Theorem 3 hold.

Theorem 8. Let $A$ be a strong Morishima matrix, i. e. in (1) it holds $A_{11} \geqq[0], A_{22} \geqq[0]$. Furthermore, let $A$ be indecomposable. Then, there exist a characteristic root $\lambda^{*}(A)$ such that:

i) $\lambda^{*}(A)$ is a positive and simple root of the characteristic equation of $A$.

ii) $\lambda^{*}(A) \geqq|\lambda|$, where $\lambda$ is any other characteristic value of $A$.

iii) $\lambda^{*}(A)>a_{i i}, \forall i=1, \ldots, n$.

iv) There exists a vector $x^{*}>[0]$ such that $I^{*} x^{*}$ is a characteristic vector of $A$ associated to $\lambda^{*}(A)$, where $I^{*}$ is the matrix appearing in the similarity transformation (2).

$v$ ) If and only if $\mu>\lambda^{*}(A)$, the matrix $[\mu I-A]^{-1}$ exists and its diagonal blocks, corresponding to the submatrices $A_{11}$ and $A_{22}$ of $A$, are positive, whereas the blocks corresponding to the submatrices $A_{12}$ and $A_{21}$ of $A$ are negative.

Other generalized Metzlerian matrices will be considered in Section 3 of the present paper. We point out that still other generalizations of the Metzlerian case, important in the analysis of stability of dynamic economic systems, were considered by Mukherji (1972), Quirk (1974), Sato (1972, 1973).

\section{Generalizations of Ohyama's Results}


One of the most interesting attempts to enlarge the class of the Metzlerian assumptions in the study of stability and comparative statics for a dynamic economic system, is due to Ohyama (1972). We briefly describe the Walrasiantype model considered by Ohyama and the results this author has obtained. Then, we shall generalize these results.

We are given an economic system where $(n+1)$ commodities are exchanged, commodities labelled $0,1, \ldots, n$. The respective aggregate excess demand functions are denoted by $E_{0}, E_{1}, \ldots, E_{n}$; these functions depend from a positive vector of prices

$$
P=\left[P_{0}, P_{1}, \ldots, P_{n}\right]^{\top}>[0]
$$

and from a vector of independent parameters (or shift parameters or exogenous variables)

$$
z=\left[z_{1}, z_{2}, \ldots, z_{m}\right]^{\top},
$$

vector belonging to a set $Z \subseteq \mathbb{R}^{m}$.

Let us suppose that the functions

$$
E_{i}=E_{i}(P, z), i=0,1, \ldots, n
$$

satisfy the following properties:

I) Every function $E_{i}$ is continuously differentiable with respect to $(P, z)$.

II) Walras' Law is assumed to hold:

$$
P>[0] \Longrightarrow \sum_{i=0}^{n} P_{i} E_{i}(P, z)=0 .
$$

III) Every function $E_{i}$ is positively homogeneous of degree zero with respect to $P$ :

$$
\lambda>0, P>[0] \Longrightarrow E_{i}(\lambda P, z)=E_{i}(P, z), \quad i=0,1, \ldots, n .
$$

Being $P_{0}>0$, we can consider, instead of functions (3), the corresponding functions

$$
f_{i}=f_{i}(p, z)=E_{i}\left(\frac{1}{P_{0}} P, z\right)=E_{i}(P, z), \quad i=0,1, \ldots, n,
$$

where $P_{0}$ is considered as a numéraire and $p$ is a vector of relative prices:

$$
p=\left[p_{1}, p_{2}, \ldots, p_{n}\right]^{\top}=\left[P_{1} / P_{0}, P_{2} / P_{0}, \ldots, P_{n} / P_{0}\right]^{\top}>[0] .
$$

$I V)$ Given $z=z^{*}$, with $z^{*} \in \operatorname{int}(Z)$, then there exists an equilibrium vector $P=P^{*}>[0]$ such that

$$
E_{i}\left(P^{*}, z^{*}\right)=0, \quad i=0,1, \ldots, n
$$

therefore there exists a vector of relative equilibrium prices

$$
p=p^{*}=\left[P_{1}^{*} / P_{0}^{*}, P_{2}^{*} / P_{0}^{*}, \ldots, P_{n}^{*} / P_{0}^{*}\right]^{\top}>[0]
$$

for which it holds

$$
f_{i}\left(p^{*}, z^{*}\right)=0, \quad i=0,1, \ldots, n .
$$

$V$ ) We choose the units of commodities such that all prices are equal to unity at equilibrium (without loss of generality):

$$
P_{0}^{*}=P_{1}^{*}=\ldots=P_{n}^{*}=1
$$

and therefore

$$
p^{*}=[1,1, \ldots, 1]^{\top}=u \text {. }
$$

$V I)$ If we adopt the notations

$$
f_{i j} \equiv E_{i j}\left(P^{*}, z^{*}\right), \quad E_{i j}(P, z) \equiv \partial E_{i} / \partial P_{j},
$$


the vector $p^{*}$ will be called a regular equilibrium vector if the matrix

$$
F=\left[f_{i j}\right] \quad i, j=1,2, \ldots, n,
$$

evaluated at $\left(p^{*}, z^{*}\right)$ is non-singular: $\operatorname{det} F \neq 0$.

We note that, by (6), (7) and (9), at $(p, z)=\left(p^{*}, z^{*}\right)$, we have also $f_{i j}=\partial f_{i} / \partial p_{j}, i=0,1, \ldots, n ; j=1,2, \ldots, n$. In order to obtain $\operatorname{det} F \neq 0$ it is sufficient to assume that in the matrix $\hat{F}=\left[f_{i j}\right], i, j=0,1, \ldots, n$, singular by assumptions (4) or (5), a principal submatrix of order $n$, such as, e. g., $F$, is non-singular at $\left(p^{*}, z^{*}\right)$. This assures that also the other $n$ principal submatrices of order $n$ are non-singular and puts into evidence that the choice of the numéraire is not relevant for the "regularity" of the equilibrium point.

It is well-known that in economic analysis the matrix $F$ is called "substitution matrix". If $F$ is Metzlerian, the economic system described has the "weak gross substitutability property" at $p^{*}$ (see, e. g., Morishima (1964)). For notational convenience, let us write

$$
f_{i 0} \equiv \frac{\partial E_{i}\left(P^{*}, z^{*}\right)}{\partial P_{0}}, \quad i=0,1, \ldots, n
$$

and

$$
f_{0 j} \equiv \frac{\partial E_{0}\left(P^{*}, z^{*}\right)}{\partial P_{j}}, j=0,1, \ldots, n .
$$

As usual, the dynamic behaviour of the normalized price vector $p$ is described by a system of first-order differential equations (together with an initial condition $p(0)=p^{o}>[0]$ ) of the type

$$
p^{\prime}(t)=D F\left(p(t)-p^{*}\right), \quad t \geqq 0
$$

or of the type

$$
p^{\prime}(t)=W F\left(p(t)-p^{*}\right), \quad t \geqq 0,
$$

where $D \in \mathfrak{D}_{+}$and $W=\left[w_{i j}\right], i, j=1, \ldots, n$, denotes a positive definite (symmetric) matrix. The global asymptotic stability of the equilibrium solution $p^{*}$ of the linearized process (10) or (11) is assured if and only if $D F$ or $W F$ are stable matrices, i. e. $D F(W F)$ has all characteristic values with a negative real part.

In particular, we recall that if $D F$ is stable for any choice of $D$ in the class $\mathfrak{D}_{+}$of diagonal matrices with a positive diagonal, we say that $F$ is $D$-stable. If $W F$ is stable for any choice of $W$ in the class of (symmetric) positive definite matrices, we say that $F$ is $W$-stable.

Another interesting problem in economic analysis consists in establishing "comparative statics" results for the system

$$
f_{i}(p, z)=0, \quad i=1, \ldots, n .
$$

The comparative statics analysis of this system involves the investigation of changes in the equilibrium price vector $p^{*}$ following a change in the values taken on by the shift parameters described by vector $z$. Under assumptions $I$ )$V I)$ we can use the classical Implicit Function Theorem (see, e. g., Apostol (1974)) in order to deduce in system (12) a locally unique (vector) implicit function $p(z)$ : there exists a neighborhood $U\left(z^{*}\right)$ of $z^{*}$ such that for any $z \in U\left(z^{*}\right)$ the function $p=h(z)$ is uniquely determined and has all components continuously differentiable on $U\left(z^{*}\right)$. Therefore, if we choose $k \in\{1,2, \ldots, m\}$, from (12) we can deduce the system

$$
\sum_{j=1}^{n}\left(\partial f_{i}(p, z) / \partial p_{j}\right)\left(\partial p_{j} / \partial z_{k}\right)+\partial f_{i}(p, z) / \partial z_{k}=0, \quad i=1,2, \ldots, n,
$$

with $z \in U\left(z^{*}\right)$. In particular, keeping (6), (7) and (9) into account and using the notations of Assumption VI), we can rewrite relation (13), for $z=z^{*}$, in the form

$$
F \pi+\Phi^{k}=[0],
$$

where

$$
\left\{\begin{array}{l}
\pi=\left[\pi_{1}, \pi_{2}, \ldots, \pi_{n}\right]^{\top}=\left[\partial p_{1} / \partial z_{k}, \partial p_{2} / \partial z_{k}, \ldots, \partial p_{n} / \partial z_{k}\right]^{\top} \\
\Phi^{k}=\left[\partial f_{1} / \partial z_{k}, \partial f_{2} / \partial z_{k}, \ldots, \partial f_{n} / \partial z_{k}\right]^{\top}
\end{array}\right.
$$


for $(p, z)=\left(p^{*}, z^{*}\right)$.

Being $F$ non-singular, system (14) admits the unique solution

$$
\pi=-\left(F^{-1}\right) \Phi^{k}
$$

which may be called the fundamental relation of comparative statics (Takayama (1985)). Comparative statics information on system (15) will be available if the knowledge of the sign patterns of $F$ and $\Phi^{k}$ is sufficient to get information on the sign pattern of $\pi$.

In order to complete and generalize the results obtained by Ohyama, we introduce the following notations and definitions.

- A nonnegative square matrix $S \geqq[0]$ is a stochastic matrix if $S u=u$.

- The equilibrium price vector $p^{*}$ is:

a) a weak Metzlerian equilibrium if

$$
\text { either } F \text { is Metzlerian and } f_{i 0} \geqq 0, \forall i \in N \text { or } F \text { is Metzlerian and } f_{0 i} \geqq 0, \forall i \in N \text {. }
$$

b) a Metzlerian equilibrium if

$$
F \text { is Metzlerian and } f_{i 0}+f_{0 i}>0, \forall i \in N \text {. }
$$

c) a strongly Metzlerian equilibrium if

$$
F \text { is Metzlerian, } f_{i 0} \geqq 0, f_{0 i} \geqq 0, f_{i 0}+f_{0 i}>0, \forall i \in N .
$$

d) an equilibrium of Ohyama, of, respectively, type I and type II, if it holds

$$
f_{i 0}>0, f_{0 i}>0, \forall i \in N
$$

and there exists an $n \times n$ stochastic definite positive matrix $S$ such that, respectively, we have

$$
S F \text { is Metzlerian }
$$

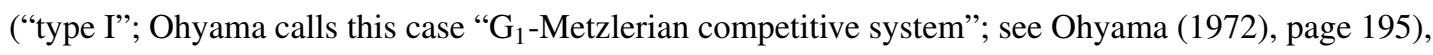

$$
S\left(F+F^{\top}\right) \text { is Metzlerian }
$$

("type II"; Ohyama calls this case " $\mathrm{G}_{2}$-Metzlerian competitive system").

e) an equilibrium of Nikaido if it is of Ohyama of type II, with $S=I$.

f) an equilibrium of Morishima generalized (in the sense of Ohyama), if $N$ can be partitioned into several subsets, disjoint and non-empty, $N_{1}, N_{2}, \ldots, N_{h}$, such that every matrix of the type $\left[f_{i j}\right], i, j \in N_{r}, r=1,2, \ldots, h$, is Metzlerian and if $i$ and $j$ belong to distinct subsets, then it holds $f_{i j} \leqq 0$. (The definition considered by Ohyama is not complete and is stronger).

g) an equilibrium of Morishima, if in $f$ ) we have $h=2$.

Always in order to generalize the results of Ohyama, we introduce also the following definitions.

Definition 4. The matrix $F=\left[f_{i j}\right], i, j=1,2, \ldots, n$, is called

i) quasi-Metzlerian of type $O\left(F \in Q_{0}\right)$ if there exists a vector $q>[0]$ and a positive definite matrix $A$ such that

$$
\begin{gathered}
\left(Q+Q^{\top}\right) \text { is Metzlerian } \\
\left(Q+Q^{\top}\right) q<[0],
\end{gathered}
$$

being $Q=A F$. 
ii) quasi-Metzlerian of type $1\left(F \in Q_{1}\right)$ if $F \in Q_{0}$ and, moreover, $A \geq[0]$ and $Q$ is Metzlerian.

iii) quasi-Metzlerian of type $2\left(F \in Q_{2}\right)$ if $F \in Q_{0}$, but with $Q=A\left(F+F^{\top}\right)$.

The results obtained by Ohyama can be synthetized as follows (here $\pi$ is the solution (15) of system (14)).

R.I) If $p^{*}$ is an equilibrium of Ohyama of type I, then $F$ is stable and imperfectly Hicksian; moreover, with $\Phi^{k}=u^{k}$, it holds $\pi \geq[0], \pi_{k}>0$.

R.II) If $p^{*}$ is an equilibrium of Ohyama of type II, then $F$ is $D$-stable and Hicksian; moreover, with $\Phi^{k}=u^{k}$, it holds $\pi_{k}>0$.

R.III) If $p^{*}$ is strongly Metzlerian and relations (19) hold, then $F$ is $D$-stable and Hicksian; moreover, with $\Phi^{k}=u^{k}$, it holds $\pi \geq[0], \pi_{k}>0$.

R.IV) If $p^{*}$ is an equilibrium of Nikaido, then $F$ is $D$-stable and Hicksian; moreover, with $\Phi^{k}=u^{k}$, it holds $\pi_{k}>0$.

$R . V)$ If $p^{*}$ is an equilibrium of Morishima generalized and it holds

$$
f_{i 0}+2 \sum_{j \notin N_{r}} f_{i j}>0, \quad \forall i \in N_{r}, \quad \forall r=1,2, \ldots, h,
$$

then $F$ is $D$-stable.

The following theorems generalize the results $R . I)-R . V$ ) of Ohyama.

Theorem 9. If $F \in Q_{0}$, then $F$ is stable and with $\Phi^{k}=u^{k}$, we have $\left(a_{k 1} \pi_{1}+\ldots+a_{k n} \pi_{n}\right)>0$.

Proof. From (23) we get, being $Q=A F$,

$$
0>\left(Q+Q^{\top}\right)_{i} q=2 q_{i i} q_{i}+\sum_{\substack{j \in N \\ j \neq i}}\left(q_{i j}+q_{j i}\right) q_{j}, \forall i \in N .
$$

This implies, being $q>[0]$, and by relation (22), that $\left(Q+Q^{\top}\right)$ has a negative principal diagonal:

$$
q_{i i}<0, \forall i \in N \text {. }
$$

From (24) we have also

$$
-2 q_{i i} q_{i}>\sum_{\substack{j \in N \\ j \neq i}}\left(q_{i j}+q_{j i}\right) q_{j}, \forall i \in N,
$$

that is, taking (25) and (22) into account,

$$
q_{i}\left|q_{i i}+q_{i i}\right|>\sum_{\substack{j \in N \\ j \neq i}}\left(q_{i j}+q_{j i}\right) q_{j}, \forall i \in N
$$

Being $q>$ [0], relations (25) and (26) put into evidence that $\left(Q+Q^{\top}\right)$ has a negative dominant diagonal; therefore the matrix $\left(Q+Q^{\top}\right)=A F+F^{\top} A$ is stable (see Theorem 2) and negative definite, being symmetric. Therefore, being $A$ definite positive, $F$ is stable (Theorem 2). Then, we note that, being $\operatorname{det} F \neq 0$ and taking the assumptions made on $A$ and $Q$ into account, with $\Phi^{k}=u^{k}$, system (14) admits the representation

$$
F \pi=-u^{k} ; Q \pi=-A^{k}
$$

and it admits the unique solution

$$
\pi=-F^{-1} u^{k}=-\left(F^{-1} A^{-1}\right) A u^{k}=-Q^{-1} A^{k},
$$

where it holds $\pi \neq[0]$ and also, taking (27) into account and the facts that $A$ is symmetric and $Q$ is quasi-negative definite,

$$
0<-\pi^{\top} Q \pi=\pi^{\top} A^{k}=A_{k} \pi=a_{k 1}+\ldots+a_{k n} \pi_{n} .
$$


Theorem 10. If $F \in Q_{1}$, then $F$ is stable and imperfectly Hicksian; moreover, with $\Phi^{k}=u^{k}$, we have $\pi \geq[0]$, $\pi_{k}>0$.

Proof. As $F \in Q_{1}$, then $F \in Q_{0}$ and, thanks to Theorem 9, $F$ is stable, (25) holds true and $Q$ is quasi-negative definite. Therefore $Q$ is stable (see Theorem 2) and it holds, being $Q$ Metzlerian, $Q^{-1} \leq[0]$ (Theorem 1). We have therefore, being $\operatorname{det} F \neq 0, A \geq[0]$ and $Q=A F$,

$$
[0] A=[0] \geq Q^{-1} A^{-1} A=F^{-1},
$$

that is

$$
f_{i i}^{-1}=\left(F_{i i} / \operatorname{det} F\right) \leqq 0, \forall i \in N .
$$

From $Q=A F$ we have $A=A F F^{-1}=Q F^{-1}$ and therefore

$$
a_{i i}=Q_{i}\left(F^{-1}\right)^{i}=q_{i i} f_{i i}^{-1}+\sum_{\substack{j \in N \\ j \neq i}} q_{i j} f_{j i}^{-1}, \forall i \in N,
$$

that is, by virtue of (25),

$$
f_{i i}^{-1}=\left(a_{i i}-\sum_{\substack{j \in N \\ j \neq i}} q_{i j} f_{j i}^{-1}\right) / q_{i i}, \forall i \in N,
$$

The assumption that $A$ is positive definite implies $a_{i i}>0$ (if we would have $a_{i i} \leqq 0$ for some $i \in N$, for $x=u^{i}$ we would get $x^{\top} A x=a_{i i} \leqq 0$, being $x \neq[0]$ ). Therefore, being $Q$ Metzlerian and taking (29), (25) and (30) into account, it holds

$$
f_{i i}^{-1}=\left(F_{i i} / \operatorname{det} F\right)<0, \forall i \in N,
$$

so $F$ is imperfectly Hicksian. We remark that with $\Phi^{k}=u^{k}$, relations (28) still hold; from the same relations, taking (29) and (31) into account, we have

$$
\begin{gathered}
\pi=-\left(F^{-1}\right) u^{k} \geq[0], \\
\pi_{k}=-f_{k k}^{-1}>0 .
\end{gathered}
$$

Theorem 11. If $F \in Q_{2}$, then $F$ is $W$-stable and Hicksian; moreover, with $\Phi^{k}=u^{k}$, we have $\pi_{k}>0$.

Proof. Following the same lines of the proof of Theorem 9, we have that if $F \in Q_{2}$, then $\left(Q+Q^{\top}\right)=A(F+$ $\left.F^{\top}\right)+\left(F+F^{\top}\right) A$ is negative definite. Being $A$ positive definite by assumption, then $\left(F+F^{\top}\right)$ is stable (Theorem 2 ) and, being symmetric, it is also negative definite. Therefore $F$ is quasi-negative definite and also $W$-stable and Hicksian (Theorem 2). Therefore, with $\Phi^{k}=u^{k}$, in the unique solution $\pi=-\left(F^{-1}\right)^{k}$ of system (14), it holds $\pi_{k}=\left(\left(-F_{k k}\right) / \operatorname{det} F\right)>0$.

Before stating the next results, we note that, under assumptions $I)-V I$ ) of the present section, the so-called Walras and homogeinity laws in differential form hold, i. e. we have

$$
\begin{aligned}
& F^{\top} u=-\left[f_{01}, f_{02}, \ldots, f_{0 n}\right]^{\top} \\
& F u=-\left[f_{10}, f_{20}, \ldots, f_{n 0}\right]^{\top} .
\end{aligned}
$$

Relation (33) can be obtained by differentiating, with respect to $P_{j}, j \in N$, the two members of the equality appearing in (4) after the implication operation and taking (8) into account, together with the conventions made on $P^{*}, p^{*}$ and $F$ for $(P, z)=\left(P^{*}, z^{*}\right)$. The same procedure allows to get relation (34), by differentiating with respect to $\lambda$ the two expressions after the implication operation of relation (5) or also by means of the well-known Euler Theorem on differentiable homogeneous functions.

Theorem 12. If $p^{*}$ is a weak Metzlerian equilibrium point, then $F$ is $D$-stable and Hicksian; moreover, with $\Phi^{k}=u^{k}$, we have $\pi \geq[0], \pi_{k}>0$. If $p^{*}$ is a Metzlerian equilibrium point or a strong Metzlerian equilibrium point, then $F$ is also $W$-stable. 
Proof. First, let us suppose that in (16) it holds $f_{i 0} \geqq 0, \forall i \in N$. From (34) we get

$$
f_{i i} \leqq-\sum_{\substack{j \in N \\ j \neq i}} f_{i j}, \forall i \in N
$$

As $F$ is a Non-singular Metzlerian matrix, this excludes the case $F_{i}=[0]$ for some $i \in N$; therefore we have

$$
f_{i i}<0, \forall i \in N \text {. }
$$

Hence, from (35) we obtain

$$
\left|f_{i i}\right| \geqq \sum_{\substack{j \in N \\ j \neq i}}\left|f_{i j}\right|, \forall i \in N .
$$

Then, we remark that if $\lambda$ is a characteristic value of $F$, with $\operatorname{Re}(\lambda) \geqq 0$, given the matrix $K=(F-\lambda I)=\left[k_{i j}\right]$, we have, by (36), $\left|k_{i i}\right|=\left|f_{i i}-\lambda\right| \geqq\left|f_{i i}\right|, \forall i \in N$, where the equality holds only if $\operatorname{Re}(\lambda)=\operatorname{Im}(\lambda)=0$, which is excluded by the assumption of the non-singularity of $F$. Therefore, with $\operatorname{Re}(\lambda) \geqq 0$, from relation (37) we have

$$
\left|k_{i i}\right|>\left|f_{i i}\right| \geqq \sum_{\substack{j \in N \\ j \neq i}}\left|f_{i j}\right|=\sum_{\substack{j \in N \\ j \neq i}}\left|k_{i j}\right|, \forall i \in N .
$$

Therefore, the matrix $K$ has a dominant diagonal and hence $\operatorname{det} K=\operatorname{det}(F-\lambda I) \neq 0$, so, with $\operatorname{Re}(\lambda) \geqq 0$, we have that $\lambda$ is not a characteristic value of $F$. Therefore, every characteristic value of $F$ has a negative real part, hence $F$ is stable. But being $F$ a Metzlerian matrix, we have (see Theorem 1) $F^{-1} \leq[0], F$ has a negative dominant diagonal, $F$ is $D$-stable and Hicksian. Under the assumption $\Phi^{k}=u^{k}$, this allows us to deduce from (15) the relations (31) and (32). In the same way we perform the proof under the assumption $f_{0 i} \geqq 0, \forall i \in N$.

If $p^{*}$ is a Metzlerian equilibrium point or also a strong Metzlerian equilibrium point, then $F$ belongs both to $Q_{1}$ and to $Q_{2}$ (it is sufficient to choose, in the relations of Definition 4, $q=u, A=I$ and take (33)-(34) into account, together with assumptions (17) or (18)). From Theorems 10 and 11 the thesis follows.

Theorem 13. If $p^{*}$ is an equilibrium of Nikaido, then $F$ is $W$-stable and Hicksian; moreover, with $\Phi^{k}=u^{k}$ we have $\pi_{k}>0$.

Proof. By performing the proof as in Theorem 12, we obtain that if $p^{*}$ is an equilibrium of Nikaido, then $F \in Q_{0}$ and $F \in Q_{2}$. From Theorem 11 we obtain the thesis of the present theorem.

Theorem 14. Let $p^{*}$ be an equilibrium of Morishima. Then, $F$ is $D$-stable and Hicksian if

$$
f_{i 0}+2 \sum_{\substack{j \in N \\ j \notin N_{r}}} f_{i j} \geqq 0, \forall r \in\{1,2, \ldots, h\}
$$

and any one of the following conditions holds:

a) $h=2$;

b) $h>2, F$ is indecomposable but (38) holds with the strict sign for at least one index $i \in L$, for any subset $L \neq \varnothing$ of $N$, such that

$$
(i \in L, j \notin L) \Longrightarrow f_{i j}=0 \text {. }
$$

Proof. From relation (38) we have, taking (34) into account,

$$
-f_{i i} \leqq \sum_{\substack{j \in N_{j} \\ j \in N_{r}}} f_{i j}-\sum_{\substack{j \in N \\ j \notin N_{r}}} f_{i j}, \forall i \in N_{r}, \forall r \in\{1,2, \ldots, h\},
$$

the inequality being strict for the values of $i$ specified in the assumptions $a$ ), $b$ ), $c$ ) of the present theorem. We build the matrix $G=\left[g_{i j}\right]$, with $g_{i j}= \pm f_{i j}$, according to the fact that $i$ and $j$ both belong or both do not belong to only one of the sets $N_{1}, N_{2}, \ldots, N_{h}$. We point out that $G$ is a Metzlerian matrix. 
From (39), from the definition of a Morishima equilibrium, the non-singularity assumption on $F$ (which excludes zero rows in $F$ ) and from the equivalence between the decomposability (if it holds) of $F$ and $G$, we obtain

$$
\begin{gathered}
g_{i i}<0, \forall i \in N \\
\left|g_{i i}\right| \geqq \sum_{\substack{j \in N \\
j \neq i}}\left|g_{i j}\right| \forall i \in N,
\end{gathered}
$$

where the inequality in (41) holds in the same way as in (39).

Now we prove that $\operatorname{det} G \neq 0$. For the case $a$ ), it is sufficient to build the matrix

$$
H=\left[h_{i j}\right], \text { with } h_{i j}=\left\{\begin{array}{l}
1, \text { if } i=j, i, j \in N_{1} \\
-1, \text { if } i=j, i, j \in N_{2} \\
0, \text { in all the remaining cases. }
\end{array}\right.
$$

Then, to remark, being $G=H F H^{-1}=H F H$, that $G$ and $F$ are similar and therefore, being $F$ non-singular, it holds $\operatorname{det} G \neq 0$.

For the cases $b$ ) and $c$ ) it is sufficient to note that $G$ has a row dominant diagonal (with $D=I$ ). Therefore, $\operatorname{det} G \neq 0$, hence $G$ has no zero characteristic values. If $\lambda$ is a characteristic value of $G$, with $\operatorname{Re}(\lambda) \geqq 0$, by (40) it will hold

$$
\left|g_{i i}-\lambda\right|>\left|g_{i i}\right|, \forall i \in N \text {. }
$$

It is therefore evident, by (41), that $(G-\lambda I)$ has a dominant diagonal, hence $\operatorname{det}(G-\lambda I) \neq 0$. It follows that every characteristic value of $G$ has a negative real part, i. e. $G$ is stable. But, being $G$ a Metzlerian matrix, then $G$ has a negative dominant diagonal (see Theorem 3). This property holds also for $F$ (being $f_{i i}=g_{i i}$ and, with $i \neq j$, $\left.\left|f_{i j}\right|=\left|g_{i j}\right|\right)$; therefore $F$ is $D$-stable and Hicksian (see Theorem 2).

It is evident that the results of Theorems 12,13 and 14 are more general than the corresponding results $R . I I I$ ), R.IV) and R.V) of Ohyama. Now we prove that the results R.I) and R.II) of Ohyama are particular cases of, respectively, Theorem 10 and Theorem 11 .

Theorem 15. The results R.I) and R.II) of Ohyama can be deduced, respectively, from Theorem 10 and Theorem 11.

Proof. First we note that in every equilibrium of Ohyama relation (19) holds; that is, taking (33) and (34) into account, we have

$$
\begin{gathered}
F^{\top} u<[0] \\
F u<[0]
\end{gathered}
$$

and moreover, in (20) and (21) the matrix $S$ is a stochastic positive definite matrix. This ensures that in every equilibrium of Ohyama it holds

$$
F^{\top} S u<[0], F S u<[0], S F^{\top} u<[0], S F u<[0] .
$$

Indeed, from the assumption $S=S^{\top}$, with $S$ a stochastic matrix, we have $S u=u$ and from, respectively, (42) and (43) we obtain

$$
\begin{aligned}
F^{\top} S u & =F^{\top}(S u)=F^{\top} u<[0], \\
F S u & =F(S u)=F u<[0],
\end{aligned}
$$

that is, the first two relations of (44). In a similar way, recalling that $S$ has all semipositive rows, it is possible to prove the last two relations of (44), i. e.

$$
S F^{\top} u<S[0]=[0] ; S F u<S[0]=[0] .
$$

It is therefore evident, with the choice $q=u, A=S$, that the relations $q>[0], A \geq[0], A$ positive definite, hold true, and in the results $R . I), R . I I)$ of Ohyama the corresponding matrices $Q=S F$ and $Q=S\left(F+F^{\top}\right)$, defined by $\left.i\right)$ and $i$ ) of Definition 3, verify, by virtue of (44), relation (23). Moreover, by (20) and (21), $Q$ is a Metzlerian matrix 
and hence also relation (22) is fulfilled. Therefore, the results $R . I$ ) and $R . I I$ ) are particular cases of, respectively, Theorem 10 and Theorem 11.

\section{Conclusions}

In the second part of the present note we have obtained some results of stability and comparative statics for a Walrasian-type model, under quantitative assumptions more general than the ones usually considered in the literature for these types of investigations. Our approach allows the presence of complementarity commodities $\left(f_{i j}<0\right.$, $i \neq j)$, weak complementarity commodities $\left(f_{i j} \leqq 0, i \neq j\right)$ ando also the existence of Giffen commodities $\left(f_{i i}=0\right)$. Moreover, also a strong "asymmetric situation" in the elements of $F$ and/or in its sign pattern, is tolerated.

Finally, we remark that, as far as we know, there are very few recent papers on economic applications of Metzlerian matrices in economic theory. A paper of D. Furth (2002) is just titled: "Why are there nowadays only a few articles on matrices in economic journals?". According to this author an answer could be that most economists are no longer interested in stability of linear or linearized systems. Indeed, mathematical economics is obviously strongly influenced by the current trends of economic researches and perhaps these ones are more changeable in time than other types of scientific researches. For the reader's convenience we suggest also the useful book of Hale, Lady, Maybee and Quirk (1999). On the other hand, there are several recent papers on Metzlerain matrices (less on Morishima matrices and other generalized Metzlerian matrices), not however concerning economic models: it is sufficient, for example, to digit "Metzler matrices" in the web-site of Mathematical Reviews or Zentralblatt für Mathematik.

\section{Acknowledgement}

The authors thank an anonymous referee for his valuable suggestions.

\section{References}

Apostol, T. M. (1974). Mathematical Analysis - Second Edition, Addison Wesley, Reading, Mass.

Arrow, K. J. (1989). A “dynamic" proof of the Frobenius-Perron theorem for Metzler matrices; in Anderson, T. W:, Athreya, K. B. and Iglehart, D. L. (Eds.), Probability, Statistics and Mathematics - Papers in Honor of Samuel Karlin, Academic Press, New York, 17-26.

Arrow, K. J., \& Mc Manus, M. (1958). A note on dynamic stability, Econometrica, 26, 448-454. http://dx.doi.org/10.2307/1907624

Arrow, K. J., \& Hahn, F. H. (1971). General Competitive Analysis, Holden-Day, San Francisco.

Berman, A., \& Plemmons, R. J. (1976). Nonnegative Matrices in the Mathematical Sciences, Academic Press, New York.

Berman, A., Neumann, M., \& Stern, R. (1989), Nonnegative Matrices in Dynamical Systems, Wiley Interscience, New York.

Farina, L., \& Rinaldi, S. (2000). Positive Linear Systems: Theory and Applications, Wiley Interscience, New York. http://dx.doi.org/10.1002/9781118033029

Fiedler, M., \& Pták, V. (1962). On matrices with non-positive off-diagonal elements and positive principal minors, Czechoslovak Mathematical Journal, 12, 382-400.

Furth, D. (2002). Why are there nowadays only a few articles on matrices in economic journals?. In Hommes, C. H., Ramer, R., and Withagen. C. A. (Eds.). Equilibrium, Markets and Dynamics - Essays in Honour of Claus Weddepohl, Springer, New York, 267-281. http://dx.doi.org/10.1007/978-3-642-56131-3_19

Gandolfo, G. (2010). Economic Dynamics, Springer, New York.

Giorgi, G. (2003). Stable and related matrices in economic theory, Control and Cybernetics, 32, 397-410.

Hale, D., Lady, G., Maybee, J., \& Quirk, J. (1999). Nonparametric Comparative Statics and Stability, Princeton Univ. Press, Princeton.

Hershkowitz, D. (1992). Recent directions in matrix stability, Linear Algebra and Its Appl., 171, $161-186$. http://dx.doi.org/10.1016/0024-3795(92)90257-B

Karlin, S. (1959). Mathematical Methods and Theory in Games, Programming and Economis, Vol. I, AddisonWesley Publ. Co., Reading, Mass. 
Kemp, M. K., \& Kimura, Y. (1978). Introduction to Mathematical Economics, Springer-Verlag, New York. http://dx.doi.org/10.1007/978-1-4612-6278-7

Magnani, U., \& Meriggi, M. R. (1981). Characterizations of $\mathcal{K}$-matrices; in Castellani, G., \& Mazzoleni, P. (Eds.). Mathematical Programming and Its Economic Applications, F. Angeli, Milan, 535-547.

Mc Kenzie, L. W. (1960). Matrices with dominant diagonals and economic theory; in Arrow, K. J., Karlin, S., \& Suppes, P. (Eds.). Mathematical Methods in the Social Sciences, Stanford Univ. Press, Stanford, Ca.

Mc Kenzie, L. W. (2002). Classical General Equilibrium Theory, The MIT Press, Cambridge, Mass..

Metzler, L. A. (1945). Stability of multiple markets: the Hicks conditions, Econometrica, 13, $277-292$. http://dx.doi.org/10.2307/1906922

Minc, H. (1988). Nonnegative Matrices, J. Wiley, New York.

Morishima, M. (1952). On the laws of change of the price-system in an economy which contains complementary commodities, Osaka Economic Papers, 1, 101-113.

Morishima, M. (1964). Equilibrium, Stability and Growth, Clarendon Press, Oxford.

Morishima, M. (1970). A generalization of the gross substitute system, Rev. of Econ. Studies, 37, $177-186$. http://dx.doi.org/10.2307/2296411

Mosak, J. L. (1944). General Equilibrium Theory in International Trade (Cowles Commission Monograph 7). The Principia Press, Bloomington, Indiana.

Mukherji, A. (1972). On complementarity and stability, J. of Econ. Theory, 4, 442-457. http://dx.doi.org/10.1016/0022-0531(72)90132-9

Murata, Y. (1977). Mathematics for Stability and Optimization of Economic Systems, Academic Press, New York.

Newman, P. K. (1959). Some notes on stability conditions, Rev. of Econ. Studies, 27, 1-9. http://dx.doi.org/10.2307/2296045

Nikaido, H. (1968). Convex Structures and Economic Theory, Academic Press, New York.

Ohyama, M. (1972). On the stability of generalized Metzlerian systems, Rev. of Econ. Studies, 39, $193-203$. http://dx.doi.org/10.2307/2296871

Plemmons, R. J. (1977). M-matrix characterizations I - Nonsingular $\mathcal{M}$-matrices, Linear Algebra and Its Appl., 18, 175-188. http://dx.doi.org/10.1016/0024-3795(77)90073-8

Poole, G., \& Boullion, T. (1974). A survey on $\mathcal{M}$-matrices, SIAM Review, 16, 419-427. http://dx.doi.org/10.1137/1016079

Quirk. J. P. (1974). A class of generalized Metzlerian matrices; in Horwich, G., \& Samuelson P. A. (Eds.). Trade, Stability and Macroeconomics: Essays in Honor of Lloyd A. Metzler, Academic Press, New York, 203-220. http://dx.doi.org/10.1016/B978-0-12-356750-5.50013-X

Sato, R. (1972). The stability of competitive system which contains gross complements, Rev. of Econ. Studies, 39, 495-499. http://dx.doi.org/10.2307/2296518

Sato, R. (1973). On the stability properties of dynamic economic systems, Int. Econ. Review, 14, $753-764$. http://dx.doi.org/10.2307/2525985

Takayama, A. (1985). Mathematical Economics, Cambridge Univ. Press, Cambridge.

Uekawa, Y. (1971). Generalization of the Stolper-Samuelson theorem, Econometrica, 39, 197-217. http://dx.doi.org/10.2307/1913341

Woods, J. E. (1978). Mathematical Economics, Longman, London.

\section{Copyrights}

Copyright for this article is retained by the author(s). with first publication rights granted to the journal.

This is an open-access article distributed under the terms and conditions of the Creative Commons Attribution license (http://creativecommons.org/licenses/by/3.0/). 